\title{
Univentricular Heart with two separate Atrioventricular Valves: Diagnosed clinically and by Trans Thoracic Two - Dimensional Echocardiography.
}

\author{
Dr. J.Rajendra Kumar ${ }^{1}$, Dr. Mamta B. Kumbhare ${ }^{2}$, Dr. P. Rohan Reddy ${ }^{3}$, \\ Dr.K. Kishore Kumar ${ }^{4}$, Lt. Col. (Dr.) Sumanta Mandal ${ }^{5}$ \\ (1 Associate Professor, 3 and 4 PG students, Department of Medicine CAIMS, Bommakal, Karimnagar (A.P.) \\ India - 505 001) \\ (2 Senior Specialist Anesthesia, Dhanvanthari Hospital, NTPC, RSTPS, Jyothi Nagar District Karimnagar (AP) \\ India-505 215) \\ (5 Physician, Military Hospital Shillong, 793001)
}

\begin{abstract}
A Univentricular Heart (UVH) is a rare congenital heart malformation. Prognosis without surgical intervention is considered to be poor and survival into the adulthood is unusual $(1,2)$. Its incidence being $1 \%$ to $4.3 \%$ of all congenital heart defects. Here we reported this rare congenital heart defect; hence it was worth reporting this interesting case.
\end{abstract}

Key Words: Univentricular Heart, Double Inlet Ventricle, Single Ventricle, TGA.

\section{Introduction:}

The term "Univentricular" refer to heart in which one ventricular chamber receives all of the flow from situs solitus, right and left atria, both of which, with the entire atrioventricular junction, are related to the single ventricle. It is also known as single ventricle or double inlet ventricle. In this condition both atria are connected with one ventricle, the AV (Atrioventricular) valves are either two, both patent and separate or there is a common AV valve without straddling or over-riding. However in majority of cases, another hypo plastic ventricular outflow chamber exists.

\section{Case Report:}

A 9 year old female patient was referred to our department (Department of medicine, Chalmeda Anand Rao Institue of Medical Science Karimnagar, A.P.), for evaluation of cyanosis and systolic murmur over the chest. As per her parents she developed cyanosis shortly after birth and a congenital heart defect was suspected. She was treated by some local private practitioners but they never consulted any specialist. Growth and developments during infancy and childhood were considered normal. She also had history of fatigue and gradual onset dyspnea but since last 6 months her exercise tolerance severely decreased, cyanosis increased and now she had grade III dyspnea. There was no history of fever, convulsion, neurological deficit, blood mixed sputum and differential cyanosis. Her physical examination revealed moderately nourished, average built, moderate cyanosis and grade I clubbing of digits (figure no.6). Hydration status was balanced. The Jugular venous pressure appeared normal. Her pulse was small in volume, slow rising and all Peripheral arterial pulses were present. No radio femoral delay. At rest her blood pressure $110 / 70 \mathrm{mmHg}$ and heart rate was 138 beats per minute. Bilateral basal crackles were present. The liver and spleen were not enlarged and there was no peripheral edema. The apex of ventricle was displaced inferiorly and lateral to the mid clavicular line. A systolic thrill was palpable in right second intercostal space and it was radiating to both carotid arteries and suprasternal notch. S1 was normal. S2 diminished and reverse split of S2 was present (P2-A2). S4 was present. A rough, ejection systolic, grade V murmur was present in aortic area. Murmur was radiating to the carotid arteries. Grade III systolic murmur was also heard over right AV (tricuspid area) and left AV (mitral area) area. Chest radiograph in postero anterior (PA) view shows cardiomegaly (cardiothoracic ratio 0.60 ), with normal pulmonary vascularity (figure no 8 ). Her arterial oxygen saturation $84 \%$ which was measured by non invasive monitoring technique (Pulse oximetry). The hemoglobin level was $16.2 \mathrm{~g} / \mathrm{dl}$, hematocrit $56 \%$, and mean corpuscular hemoglobin $31.2 \mathrm{pg}$. Serum iron $(180 \mu \mathrm{g} / \mathrm{dl})$, Transferrin $(2.5 \mathrm{~g} / \mathrm{l})$ and ferritin level $(114 \mathrm{ng} / \mathrm{ml})$ were within normal limits. Her Electrocardiogram shows, regular rhythm, heart rate 138/minute, normal PR interval (140ms), QRS duration 98ms, QTC 443ms, QRS axis - 79, "P" wave axis - 100,T wave, axis -99, Prominent "R" wave from V1 to V3, and deep 'S' and small ' $r$ ' wave from V4 to V6 (figure no 7)

The Trans Thoracic Two -dimensional echocardiography, color flow Doppler, M-Mode and spectral Doppler was done by using different windows. Her Trans Thoracic Two -Dimensional Echocardiography (TTE) 
finding includes situs solitus of viscera and atria; normal systemic and pulmonary venous connection; two separate atrioventricular valves connected to a single ventricle that is morphologically left ventricular type (figure no1, 2, 3, 4 \&5). Ventriculoarterial discordance was present (figure no 3, $4 \& 5$ ). A left sided aorta was arising from a small right ventricular outlet chamber (O.C.) with severe sub aortic obstruction (Table no 4). Outlet chamber and aorta was located anteriorly and left to the pulmonary artery (Figure no 3, 4 \&5). The aortic root was with in normal limits. Continuous wave Doppler (CWD) echocardiography across outlet chamber shows severe sub aortic stenosis (Table no 4). A right sided pulmonary artery was connected to morphological left ventricle without obstruction (Figure no 3, 4 \&5). Aortic and pulmonary valves were normal (Figure no 3). Continuous wave Doppler echocardiography at left (Mitral valve) and right ( Tricuspid valve) AV valve shows, moderate left and mild to moderate right $\mathrm{AV}$ valves regurgitation (Table no 4). Both AV valves were normal in thickness, pliable and none calcified (figure no $1 \& 2$ ). The Inferior vena cava and hepatic veins were normal. Morphological Single ventricle appeared hypertrophied and dilated in AP4C view (Figure no.1, 2 \&4). M-Mode echocardiography shows decreased ejection fraction (EF $45 \%$ in our case). We advised cardiac catheterization, and surgical correction but her parents did not agreed.

\section{Discussion:}

UVH is rare congenital anomaly occurring in $1 \%$ to $2 \%$ of all congenital defects in newborns (7). The type of presentation and natural survival dependent principally upon the pulmonary vascular resistance; upon the absence, presence and degree of pulmonary stenosis, and upon the presence, degree and progression of sub aortic stenosis (Table no 3).In patient with single ventricle of left ventricular morphology $50 \%$ are dead within 14 years with an annual attrition rate of $4.8 \%(10)$. Single ventricles of right ventricular morphology have an ever more grim natural history, with $50 \%$ survival for four years. Echo-Doppler evaluation should provide detailed information (Table no. 1). The AV connection can be different type in UVH (Table no. 2). Different nomenclatures exist like, Single ventricle and double inlet ventricle. Depending on the ventricular morphology the single ventricle can be subdivided into three types $(1,2)$, \{I $\}$ The commonest variety is in which the main ventricular morphology is the left type and the small outflow chamber is the right ventricle, this type is seen in $61 \%-80 \%$ of ceases. $\{\mathrm{II}\}$ Double inlet right ventricle with a rudimentary left ventricular outflow $\{\mathrm{III}\}$ The indeterminate type in which the ventricular morphology is not clear is present in $3-19 \%$ of cases. There may be situs solitus, inversus or ambiguous along with mesocardia or dextrocardia with each variety. Hearts with very large ventricular septal defects usually have recognizable right and left ventricular morphology in the apical position and are not considered as having univentricular AV connections. In our case TTE (AP4C and Sub costal views) and clinical features are more in favor of type I variety of UVH and situs solitus.

Generally a DILV (Double inlet left ventricle) has two separate AV valves; a common AV valve is more likely with double inlet right ventricular or indeterminate type of ventricle. Since recognition of AV valve into mitral and tricuspid may be difficult, it is better to call them as right or left AV valves. Not infrequently, this valve may be regurgitating, obstruction is much rare. In our case, TTE (in AP4C and Sub costal views) and CWD echocardiography (at AV valves); shows two separate, normal functioning AV valves and right mild to moderate and left moderate regurgitation; all these findings are more in favor of DILV. The great vessels connection being concordant in $15 \%$ and discordant (D - or- L- transposition) in $85 \%(3,9)$. In our case the great arteries connection are discordant (Figure no 3, 4 and 5). There may be double outlet to the main ventricle or rudimentary chamber or there may be just one artery arising from the ventricle as in truncus arteriosus or in cases where one of the semilunar is atretic. In double inlet right ventricle (DIRV), ventriculo-arterial connection is double outlet of the main chamber in $40 \%-100 \%$ of cases.

Double outlet type of connection is even more common in indeterminate type of UVH and seen in $85 \%$ of cases, in rest of $15 \%$, the outlet is often single (4).

Outflow tract obstruction in UVH may be pulmonary or systemic, out flow that may be valvular or subvalvular. A decrease in the size of communication between the main ventricle and rudimentary out flow chamber is also one of the causes for subvalvular outflow obstruction. Pulmonary stenosis is much more effective than pulmonary vascular resistance in achieving a favorable balance between interventricular mixing and pulmonary arterial blood flow. Survival in single ventricle with pulmonary stenosis, up to adolescence or early adulthood is not rare, and the natural history occasionally extended in to the fourth or fifth decade, with one patient reaching up to age 56 years (11).At least half a dozen women in this category have experienced relatively normal pregnancy. Subaortic stenosis has an adverse effect on survival and as the age increases outlet chamber becomes more restrictive. Subaortic stenosis has undesirable effect on the circulation of the single ventricle because pulmonary blood flow is augmented, and volume overload to the single ventricle become excessive so the survival rate and prognosis both are decreased. Sub aortic stenosis diverts even more blood into the pulmonary circulation whether or not the great arteries are transposed, and whether or not both great arteries originating from the single ventricular chamber. Pulmonary stenosis or pulmonary vascular diseases result in decline in pulmonary arterial blood flow, so the left atrium receives a smaller volume of oxygenated blood for 
delivering into single ventricle. Volume over load relieved but at the price of increased cyanosis, although streaming with in the single ventricular chamber may remain relatively good. Mild to moderate pulmonary stenosis has little adverse effect on interventricular streaming, so cyanosis remain mild, but sever pulmonary stenosis or atresia is accompanied by conspicuous cyanosis. In our case severe Subaortic obstruction was present.

Ventricular function is an important prognostic factor for immediate and long term outcome after surgery. The main ventricular chamber has to perform systolic work for both pulmonary and systemic circulation, this may result in its failure after some years (4). An early ventricular dysfunction is more likely if the main chamber is right ventricular morphology. In our case main ventricle had left ventricular morphology, so she developed late onset of LV dysfunction . Our patient had single ventricle which allow blood from right atrium (oxygen poor) to mix with blood from left atrium (oxygen rich) creating blood with an oxygen level somewhere in-between that of oxygen poor and oxygen rich blood.

UVH may be associated with atrial septal defect, patent ductus arteriosus, parachute mitral valve, anomalous systemic and pulmonary venous drainage particularly in case of situs ambiguous. In our case there was situs solitus and systemic and pulmonary venous drainage was normal.

So on the basis of clinical and by TTE findings she was diagnosed as a case of complex congenital cyanotic heart disease, Morphological LV type UVH, DILV, discordant great arteries, Sever A.S, mild to moderate right and moderate left regurgitation, and mild LVD. We advised cardiac catheterization, surgical correction and also explained prognosis without surgical correction and out come after surgery but her parents did not agreed. She was treated with diuretic, ACE inhibitors and digoxin and advised regular follow up.

\section{Treatment:}

A cardiac catheterization will usually be performed to evaluate, defects and the amount of blood that is mixing. An intravenous medication, prostaglandin is given to keep the ductus atreriosus from closing. Surgical treatment available for single ventricular physiology, discordant great arteries and sub aortic stenosis are, (1) Palliative operations - Fontan procedure, Pulmonary artery banding, Aortopulmonary shunt (In Pulmonary stenosis ) and Glenn procedure, (2) Ventricular exclusion operation , (3) Separate ventricular surgery (4) Heart transplantation (5) Damus-Keye-Stansel (DKS) operation for sub aortic stenosis.

\section{Conclusion:}

The most promising survival without surgery is seen in patient with a single ventricle of left ventricular morphology, transposition of great arteries without systemic outflow obstruction and adequately functioning AV valves and a moderate pulmonary outflow obstruction. A non invasive cardiac imaging (like echocardiography) play major role in diagnosis, assessment of ventricular function and $\mathrm{AV}$ valves, detection of complication in pre and post operative period after a palliative or definitive surgery.

\section{References:}

[1]. Anderson RH, Becker AE, Tynan M, Macartney FJ, Rigbu MZ,Wilkinson JL. The Univentricular atrioventricular correction: getting to the root of a thorny problem. Am J Cardiol 1984; 54:822-828.

[2]. Ho SY, Luberbuhler JR, Anderson RH. Pathology of heart with a univentricular atriovenrticular connection. In: Rosenberg HS, Bernstein J, eds. cardiovascular diseases .Perspectives in pediatric pathology, Volume 12, Basal: karger, 1988:66-69.

[3]. Hubta JC, Seward JB, Tajik AJ, Hoglet DJ, Edwards WD. Two Dimensional echocardiographic spectrum of univentricular atrioventricular connection. J Am Coll Cardiol 1985; 5:149-157.

[4]. Anderson RH, Tynan M, Freedom RM et.al. Ventricular morphology in the univentricular heart. Herz 1979; 4:107-112.

[5]. Samanek M. Children with congenital heart disease: Probability of natural survival .Pediatr Cardiol.1992;13:152-158

[6]. Moodie DS, Ritter DG, Tajik AJ, O'Fallon WM. Long term follow-up in the unoperated univentricular heart. Am J Cardiol. 1984; 53:1124-1128.

[7]. Samanek M, Voriskova M. Congenital heart diseases among 815,569 children born between 1980-1990 and their 15-year survival :a prospective Bohemia survival study. Pediatr Cardiol .1999; 20:411-417.

[8]. Perloff JK. The clinical recognition of congenital heart disease. Fourth edition .Philadelphia:WB.Saunders; 1994.

[9]. Van Praagh R, Ongley P, Swan H. Anatomic types of single or common ventricle in man: Morphological and geometric aspects of 60 necropsied cases. Am J Cardiol.1964:13:367-368.

[10]. Moodie D.S., Ritter D.G., Tajik A.J., and O'Fallon W.M. Long term follow-up in the unoperated univentricular heart. Am. J. Cardiol.53:1124, 1984

[11]. Mandel A., and Hirsch V.: Cor triloculare biatriatum. Report of a case with survival to the age of 29 years. Am. Hart J.66; 104, 1963. 


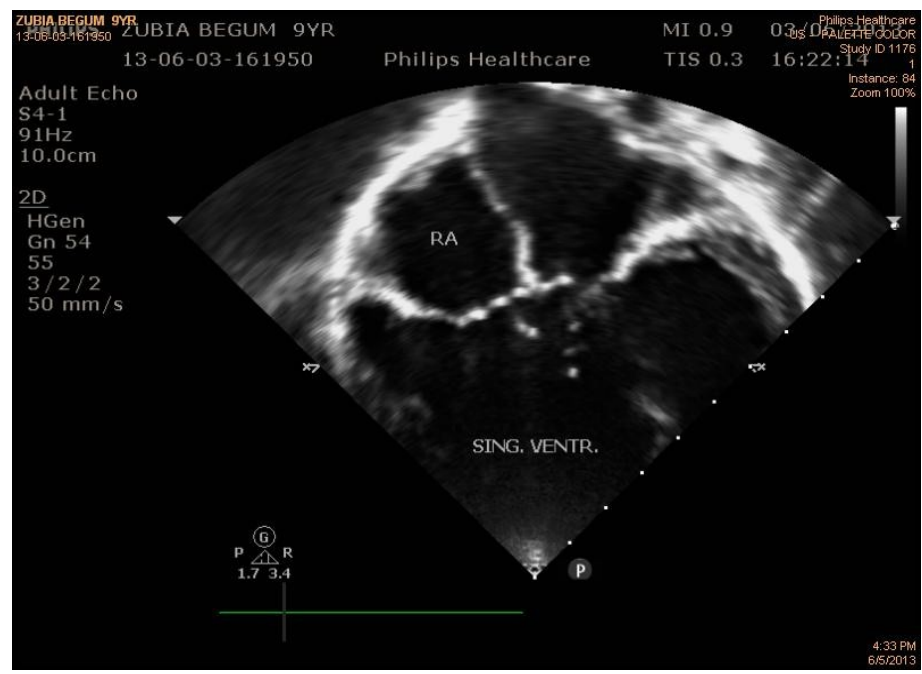

Figure no -1: TTE, AP4C view in systole shows, two separate atrium, two separate AV valves (right and left), intact inter atrial septum, morphological LV type UVH, and an outlet chamber. (RA- right atrium, Sing. Ventr -single ventricle, TTE- Tran's thoracic echocardiography, AP4C- apical 4 chamber view)

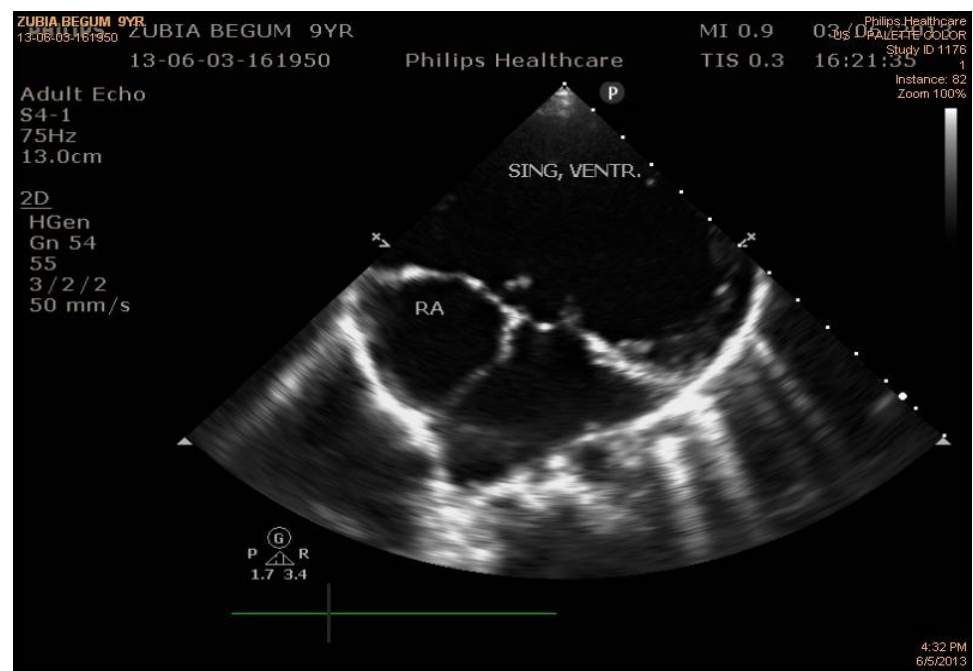

Figure no -2: TTE, AP4C view in systole shows, two separate atrium, two separate AV valves (right and left), intact inter atrial septum, morphological LV type UVH, and Hypertrophy of UVH.

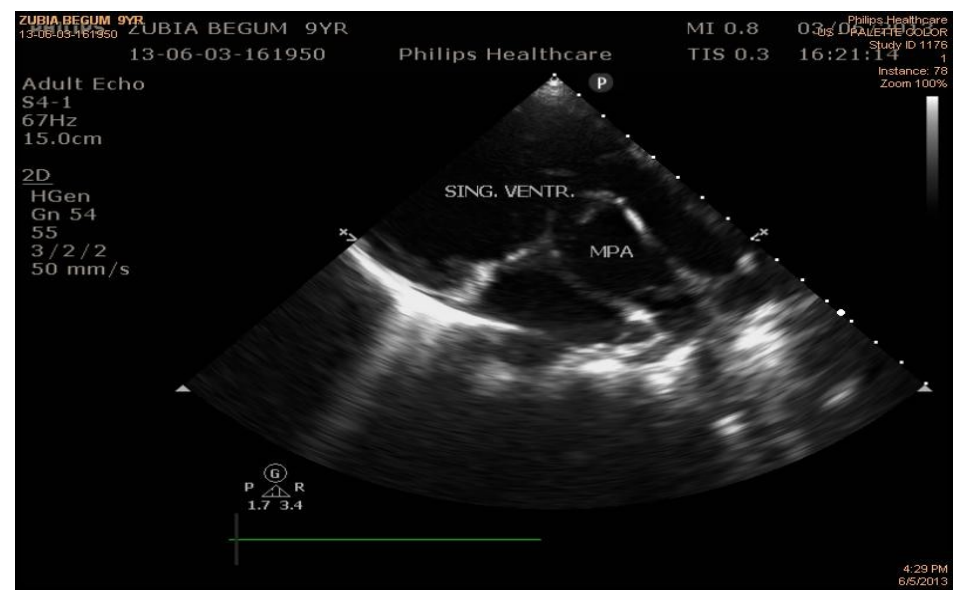

Figure no -3: TTE, PLAX view in systole shows, ventriculo arterial discordant, A left sided aorta arising from a small right ventricular outlet chamber, an aorta is anterior and left to pulmonary artery, a right sided pulmonary artery is connected to one morphological $L V$ type ventricle and two branches ( right and left) of MPA . (MPA - main pulmonary artery or pulmonary artery, PLAX- Parasternal long axis view)) 


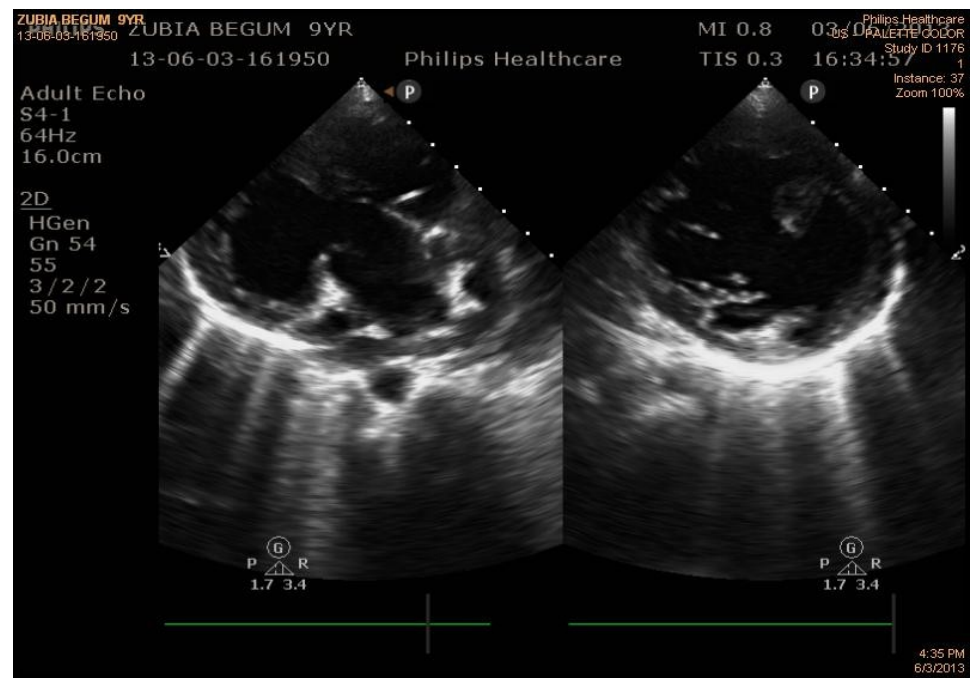

Figure no-4: (1) Right frame - PLAX shows, ventriculo arterial discordant, A left sided aorta arising from a small right ventricular outlet chamber, an aorta is anterior and left to pulmonary artery, a right sided pulmonary artery is connected to one morphological $L V$ type ventricle, two branches of MPA . (2) Left frame - PASX view shows hypertrophy of UVH. (PASX - Parasternal short axis view)

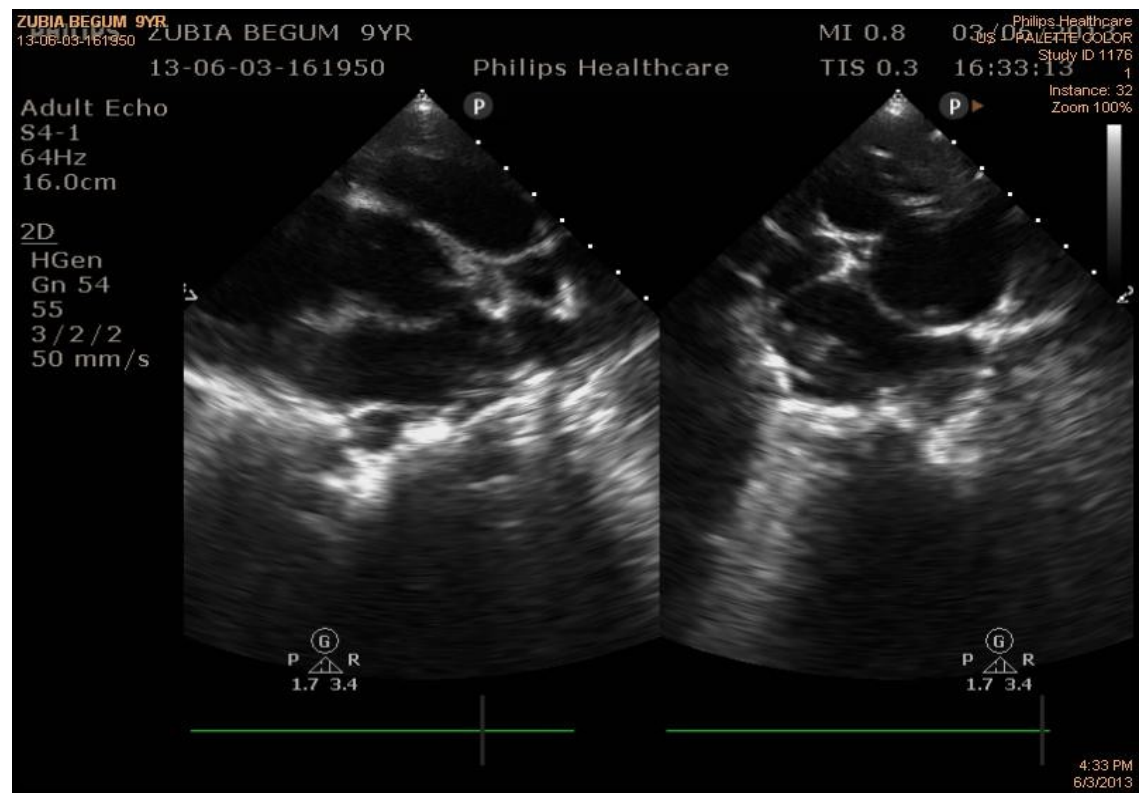

Figure no-5: Both frame - PSAX shows, A left sided aorta arising from a small right ventricular outlet chamber, an aorta is anterior and left to pulmonary artery, a right sided pulmonary artery, two braches of MPA .

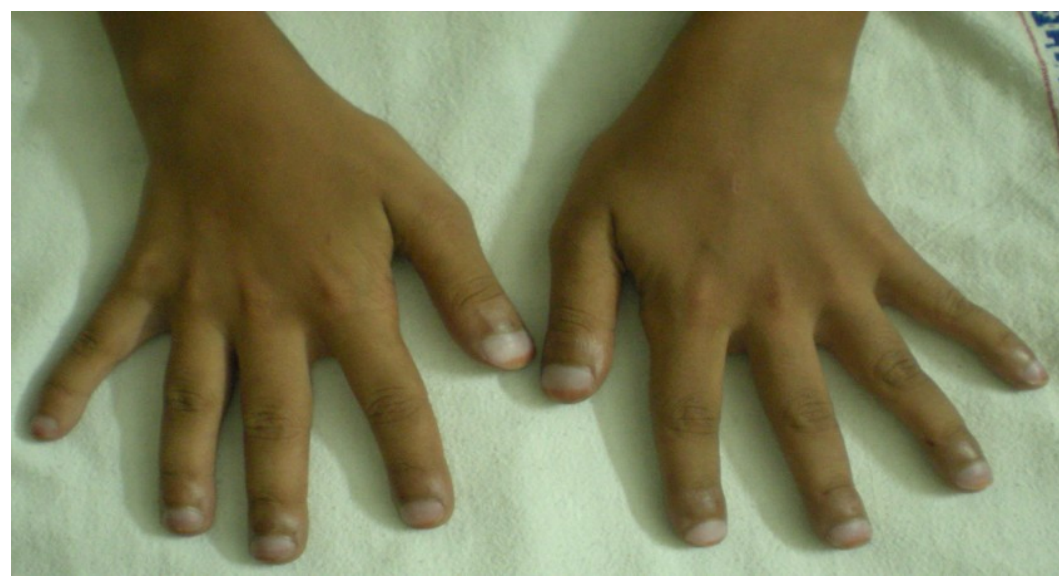

Figure no -6: Grade I clubbing of all fingers and cyanosis. 


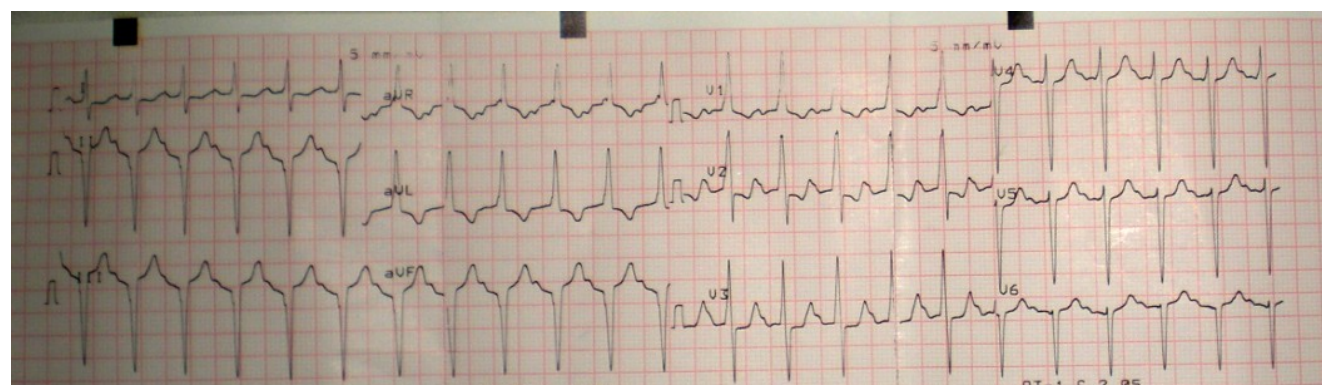

Figure no - 7: Twelve leads surface electrocardiogram shows, narrow QRS complex, normal PR interval, prominent " $\mathrm{R}$ " wave from V1 to V3, and deep 'S' and small ' $r$ ' wave from V4 to V6 chest leads.

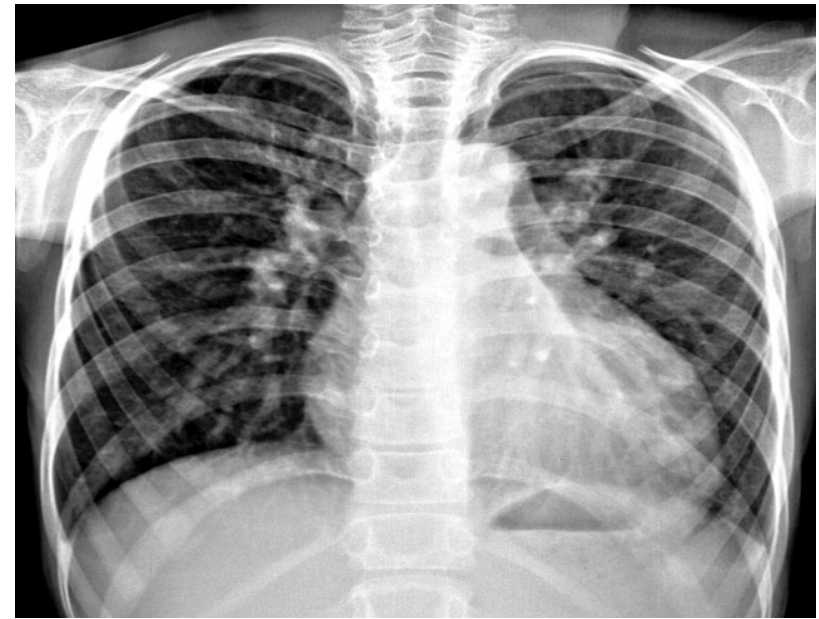

Figure no -8: X-ray chest PA view shows cardiomegaly and normal pulmonary vascularity. The large right pulmonary artery is lifted upward, causing a "Waterfall" appearance. The inverted outlet chamber (O.C.) form a bulge at the upper left cardiac border and give rise to the aorta.

Table No -1: Information provided by echo-Doppler in UVH.

1. Presence and location of outflow chamber.

2. Number and functional status of AV valves.

3. Number, position of great vessels and their relationship to the main ventricle and out flow chamber.

4. Presence and severity of out flow obstruction, mostly of pulmonary blood flow.

5. Functional performance of the main ventricle, as it is of significance in deciding the type of surgery and it determining outcome after surgery.

Table no-2: Atrio-ventricular (AV) connection in UVH.

\begin{tabular}{|l|l|}
\hline 1. & Two separate patent AV valves. \\
\hline 2. & One valve is atretic and other is patent (Classical example is tricuspid atresia). \\
\hline 3. & One common AV valve. \\
\hline 4. & Over-riding on one valve. \\
\hline 5. & Straddling of one valve. \\
\hline
\end{tabular}

Table No -3: AV connection to a single morphologic $L V$ with outlet chamber

\begin{tabular}{|l|l|}
\hline A. & $\begin{array}{c}\text { Without pulmonary stenosis } \\
1 . \quad \text { Low pulmonary vascular resistance. }\end{array}$ \\
\hline B. & With pulmonary stenosis. \\
\hline C. & With or without sub aortic stenosis. \\
\hline
\end{tabular}

Table No -4: Continuous wave Doppler echocardiography findings in our patient.

\begin{tabular}{|l|l|l|l|}
\hline Valves & $\begin{array}{l}\text { Flow velocity } \\
\text { (Meter/sec.) }\end{array}$ & $\begin{array}{l}\text { Peak pressure } \\
\text { gradient(mmHg) }\end{array}$ & $\begin{array}{l}\text { Mean pressure } \\
\text { gradient(mmHg) }\end{array}$ \\
\hline Sub Aortic valve level & 4.56 & 83.1 & 55.7 \\
\hline $\begin{array}{l}\text { Left A.V. Valve } \\
\text { (Mitral valve) }\end{array}$ & 4.67 & 87.2 & 44.2 \\
\hline $\begin{array}{l}\text { Right A.V. Valve } \\
\text { (Tricuspid valve) }\end{array}$ & 3.49 & 48.7 & 19.9 \\
\hline
\end{tabular}

